\title{
THz emissions by two-color f laments in air: Revisiting the wavelength scaling
}

\author{
L. Bergé $^{1}$, A. Nguyen ${ }^{1}$, K. J. Kaltenecker ${ }^{2}$, J. C. Delagnes ${ }^{3}$, B. Zhou ${ }^{2}$, E. Cormier ${ }^{3}$,

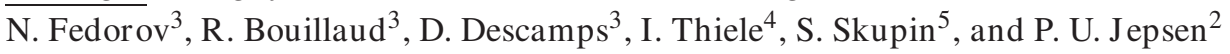 \\ ${ }^{1}$ CEA-DAM, DIF, 91297 Arpajon, France \\ ${ }^{2}$ DTU Fotonik, Department of Photonics Engineering, Technical University of Denmark, DK-2800 Kongens \\ Lyngby, Denmark \\ ${ }^{3}$ Univ. Bordeaux - CNRS - CEA, Centre Lasers Intenses et Applications, UMR 5107, 33405 Talence, France \\ ${ }^{4}$ Department of Physics, Chalmers University of Technology, SE-412 96 Göteborg, Sweden \\ ${ }^{5}$ Institut Lumière Matière, UMR 5306 Université Lyon 1 - CNRS, Université de Lyon, 69622 Villeurbanne, \\ France \\ luc.berge@cea.fr
}

\begin{abstract}
We report impressive growths in the terahertz energies supplied by air plasmas created by two-color laser pulses whose fundamental wavelength is increased. Comprehensive 3D simulations reveal the crucial role of the two-color relative phase.
\end{abstract}

OCIS codes: (320.7110) Ultrafast nonlinear optics; (350.5400) Plasmas; (260.3090) Infrared, far.

\section{Introduction}

Producing terahertz $(\mathrm{THz})$ radiation by ultrashort laser pulses has become an active $\mathrm{f}$ eld of research because of its promising applications in, e.g., spectroscopy and medical imaging [1]. Eff cient THz emitters can be obtained by focusing into air a two-color femtosecond light pulse, composed of fundamental (FH) and second (SH) harmonics, in order to create a plasma channel that acts as a frequency converter [2]. Recent studies [3, 4] showed that increasing the pump wavelength enhances the THz energy. However, there is no consensus on the gain factors expected when pushing the FH wavelength, $\lambda_{0}$, from the near-IR to the mid-IR range. Clerici et al. [3] reported THz energy yields scaling like $\lambda_{0}^{4.6}$ in the range $0.8-1.8 \propto \mathrm{m}$. By contrast, according to the local-current model $[2,4]$, the largest THz energy attained by two colors with $\pi / 2$ relative phase should follow a scaling in $\lambda_{0}^{2}$ only.

\section{Experimental campaigns vs numerical simulations}

Here we address this apparent contradiction by performing two distinct experiments scanning the wavelength range $1.2-2.6 \propto \mathrm{m}$. The experimental campaigns used two different OPA systems (TOPAS). Figure 1(a) summarizes the THz signals recorded with a pyroelectric detector. Despite the different wavelength ranges investigated, the $\mathrm{f}$ tting curves of this f gure underline impressive growths in $\lambda_{0}^{\alpha}$ with powers $\alpha$ not only exceeding $\sim 4.6$ [3], but also reaching up to almost 15 in the highest wavelength ranges. We also observe a decrease in the pyroelectric signal beyond specif c $\lambda_{0}$ values, which may indicate some optimum wavelengths where maximum THz generation is achieved. Such a behavior is present in the data points of Ref. [3] as well, recalled by the gray symbols and line.

We show that such impressive growths can be explained by changes in the laser parameters as the FH wavelength is increased [5]. Comprehensive 3D simulations combining both computations of the SH/FH energy ratio from the doubling crystal and a unidirectional numerical solver [6] are performed using the experimental values of the beam diameters and pulse durations. They reproduce reasonably well the behaviors of $\mathrm{THz}$ energies reported from the experiments, that is, not only a global growth in $\sim \lambda_{0}^{\alpha}$, but also the occurrence of optimum FH wavelengths beyond which eff ciency in THz generation drops [Fig. 1(b)]. We demonstrate that the latter effect strongly depends on the phase angle between the two colors exiting the crystal, which varies with $\lambda_{0}$ for a f xed distance separating the plasma zone from the doubling crystal. In particular, drops in the $\mathrm{THz}$ signal around specif $\mathrm{c}$ wavelengths $\left(\lambda_{0}=1.4\right.$ and $\left.2.0 \propto \mathrm{m}\right)$ are due to variations in the relative phase. A constant phase angle of $\pi / 2$ at the crystal exit renders the THz energy increase monotonous (blue curve). 


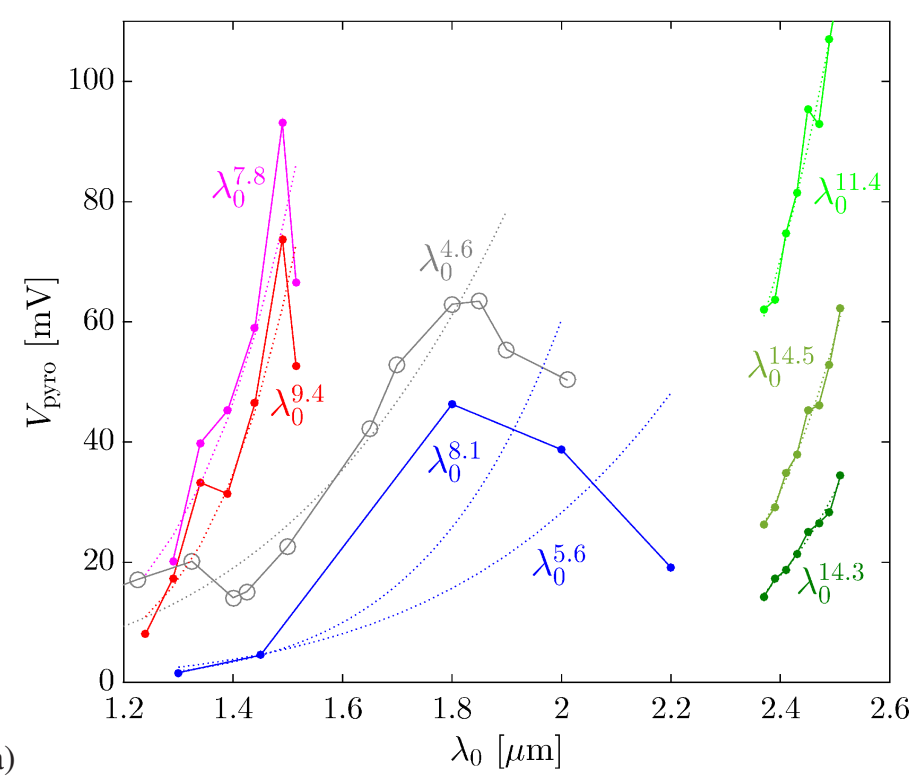

(a)

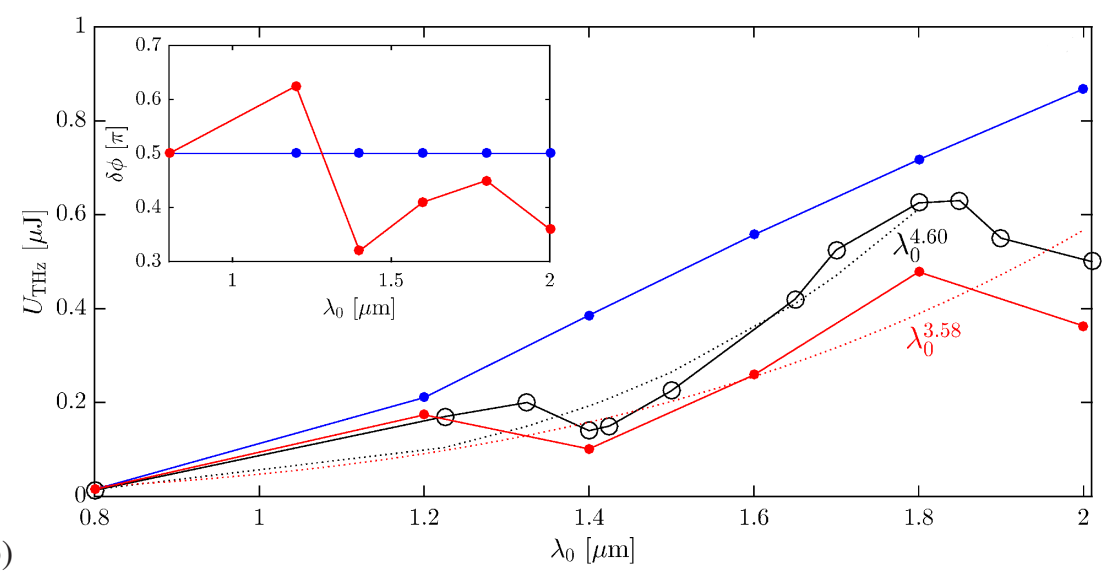

Fig. 1. (a) Pyroelectric detector signals vs FH wavelength in the frequency window $v<20 \mathrm{THz}$ for the two experimental campaigns. The average input power of the pump beam is constant for each curve, namely, 720-840 mW (red/pink curves), $300 \mathrm{~mW}$ (blue curve), 348-420-558 mW (green curves). Dotted curves are f tting curves in $\lambda^{\alpha}$. Gray circles recall the data of Ref. [3]. (b) THz energy yield computed with the 3D UPPE model (red dots) for the experimental data of [3] (black circles) with the initial phase shifts specif ed in inset. The blue curve is associated to $\pi / 2$ phase angle at the exit of the doubling crystal.

\section{References}

1. M. Tonouchi, "Cutting-edge terahertz technology," Nat. Photon. 1, 97-105 (2007).

2. K. Y. Kim, A. J. Taylor, J. H. Glownia, and G. Rodriguez, "Coherent control of terahertz supercontinuum generation in ultrafast laser-gas interactions," Nat. Photon. 2, 605-609 (2008).

3. M. Clerici, M. Peccianti, B. E. Schmidt, L. Caspani, M. Shalaby, M. Giguère, A. Lotti, A. Couairon, F. Légaré, T. Ozaki, D. Faccio, and R. Morandotti, "Wavelength scaling of terahertz generation by gas ionization,” Phys. Rev. Lett. 110, 253901 (2013).

4. A. Nguyen, P. González de Alaiza Martínez, J. Déchard, I. Thiele, I. Babushkin, S. Skupin, and L. Bergé, "Spectral dynamics of THz pulses generated by two-color laser flaments in air," Opt. Express 25, 47204740 (2017).

5. N. S. Makarov, M. Drobizhev, and A. Rebane, "Two-photon absorption standards in the 550-1600 nm excitation wavelength range," Opt. Express 16, 4029-4047 (2008).

6. M. Kolesik and J. V. Moloney, "Nonlinear optical pulse propagation simulation: From Maxwell's to unidirectional equations," Phys. Rev. E 7, 036604 (2004). 